\title{
Plasma cytokines quantification among Trypanosoma brucei rhodesiense sleeping sickness cases and controls in Rumphi, Malawi
}

\author{
Kelita Kamoto, Arthur Chiwaya, Peter Nambala, Pricilla Chammudzi, Edward Senga, John Chisi, \\ Enock Matovu, Janelisa Musaya
}

TrypanoGEN Research Group, members of The H3Africa Consortium

Correspondance: Kelita Kamoto; Email; kkelita@yahoo.com
Introduction
Trodionoma
African countries, including Malawi. For a long time, untreated HAT infections were believed to be $100 \%$ fatal. However, recent studies show that infection by T.b. rhodesiense can result in a wide range of clinical outcomes in its human host. Apart from other factors such as parasite diversity, cytokines have been strongly implicated to play a major role in the outcome of T.b. rhodesiense infections.
In this study, we quantify the levels of three cytokines Interleukin-8 (IL-8), Tumor Necrotic Factor alpha (TNF- $\alpha$ ) and Interleukin -10 (IL-10) in plasma amongst HAT cases (treated and untreated) and controls recruited during medical survey.
Methods
Two-hundred and thirty-three plasma samples (HAT cases and controls) from Rumphi, one of the endemic areas in Malawi were used. Blood collected was centrifuged, plasma extracted and stored in cryovials at $-80^{\circ} \mathrm{C}$ until processing. Plasma cytokine concentration was measured using ELISA.
Results
Plasma samples for 233 individuals, 76 HAT cases and 157 controls were quantified. Among the cases, nine had their plasma collected before treatment (untreated) and the rest were treated before blood for plasma analysis was collected. Controls had significantly higher mean plasmatic levels of TNF- $\alpha(94.5 \pm 474.12 \mathrm{pg} / \mathrm{ml})$ and IL-8 $(2258.6 \pm 5227.4 \mathrm{pg} / \mathrm{ml})$ than cases TNF- $\alpha(29.35 \pm 181.58 \mathrm{pg} / \mathrm{ml})$ and IL-8 (1191.3 $\pm 4236.09 \mathrm{pg} / \mathrm{ml})$. Controls and cases had similar mean levels of IL-10 in plasma. Only IL-8 had statistically significant higher median levels in the untreated than treated HAT cases $\mathrm{P}=0.006$.
Conclusion
Our data suggest that cytokines could be considered as biomarkers of HAT infection and treatment. Further studies with a larger cohort of cases and additional cytokines which are known to be associated with HAT infection outcomes will be required to evaluate these cytokines further.

Abstract

Keywords; Sleeping sickness, Human African Trypanosomiasis, Trypanosoma brucei rhodesiense, Plasma cytokines, IL-8, TNF- $\alpha$, IL-10

\section{Introduction}

Human African trypanosomiasis (HAT), also known as sleeping sickness, is one of the neglected tropical diseases that is endemic in sub-Saharan Africa. In the beginning of $20^{\text {th }}$ century millions of people died from the disease and available estimates showed that 36 African countries and over 60 million people were at risk of being infected ${ }^{1,2}$. HAT is caused by extra-cellular protozoan parasites Trypanosoma brucei, T.b, which is transmitted by tsetse flies of the genus Glossina. T. b. rhodesiense is the cause of sleeping sickness in the eastern and southern Africa, including Malawi. Its reservoirs are in livestock and wildlife. T.b. gambiense causes sleeping sickness in central and western Africa, and causes the majority of human cases with the main reservoir being humans ${ }^{2,3}$.

Recently there has been a reduction in the number of reported new T. b. rhodesiense HAT cases as well as deaths ${ }^{4}$. Under reporting has been associated with this reduction since many cases as high as $40 \%$ go undetected. T. $b$. rhodesiense HAT being a zoonotic disease still poses as a risk to many people earning a living in or around national parks, forest land and large water bodies ${ }^{5,6}$. There are two clinical stages for sleeping sickness, the haemolymphatic stage followed by the meningoencephalitic stage. The two subspecies have different rates of disease progression; T.b. rhodesiense infection is typically described as an acute disease with rapid progression to late stage whereas T.b. gambiense progresses more slowly ${ }^{3}$.

For a long time, untreated T.b. rhodesiense infections were believed to be $100 \%$ fatal, with death occurring within weeks or months of symptoms first appearing ${ }^{7,8}$. However, there is increasing evidence that infection by T.b. rhodesiense can result in a wide range of clinical outcomes with different severity and disease progression in different human hosts ${ }^{9-11}$

Some studies in human have demonstrated that there are difference in disease progression in individuals coming from same foci or geographically related foci ${ }^{12}$. These differences could be attributed to either parasite diversity or host immune response to infections or both. Since cytokines regulate the host response to infections, trauma, inflammations and immune responses ${ }^{13}$, it has been suggested that cytokines could be the key players in HAT disease processes ${ }^{11,12,14}$.

Once a human is infected by the African trypanosomes, innate and adaptive immune responses are activated ${ }^{15}$. Macrophages are one of the important cells involved in the innate immunity during African trypanosomiasis. Macrophages clear the parasites through phagocytosis ${ }^{16}$. The dead trypanosomes release DNA and other molecules 
that activate macrophages to secrete pro-inflammatory cytokines $/$ molecules $^{17}$.

In HAT infection, some of the known Pro-inflammatory (upregulated during inflammation) cytokines are IL-1, IL6, IL-12, TNF- $\alpha$ and IFN- $\gamma^{18-22}$ and the anti-inflammatory cytokines include type II cytokines such as IL-4, IL-10 and IL-13. These cytokines down regulate the macrophages to become more anti-inflammatory type, thus controlling both the parasites and pathology ${ }^{23}$. Studies have suggested that cytokine responses may influence the outcome of African trypanosomiasis infections ${ }^{24,25}$. It has been demonstrated that IL-10 and IL-6 are associated with the protection from neuroinflammatory pathology of $\operatorname{HAT}[26,27]$ whereas $\mathrm{TNF}_{-} \alpha$ is involved in both parasitemia control and infectionassociated pathology ${ }^{28}$. IL-10 is found to be high in CSF of late-stage patients compared to early-stage patients, because it acts as anti-inflammatory protein and B-cell stimulator. Therefore, it is suggested that CSF IL-10 could be used for staging and treatment monitoring ${ }^{29}$.

Other cytokines such as IL-8 play an important role in resistance to infection by attracting neutrophils and $\mathrm{T}$ cells to infection site $e^{30}$. In a study of T. b. gambiense patients, IL- 8 was high in both serum in the early stage and CSF in the late stage of infection and dropped after treatment ${ }^{29}$. Published data $^{31}$ has shown that gambiense sero positive individuals are able to control T. b. gambiense infection and that high levels of IL- 8 are associated with infection control whereas individuals with high levels of IL10 and TNF $\alpha$ may end up developing the disease.

\section{Methodology}

\section{Population and study design}

The survey was done from January 2014 to June 2015 at Rumphi district hospital, northern Malawi. Rumphi District has the highest prevalence of HAT cases and is regarded as the epicenter of HAT infections in Malawi ${ }^{32}$. Sleeping sickness is endemic in areas where other tropical diseases, including malaria are also common ${ }^{10}$.

Cases were identified through active and passive surveys. Hospital case files were checked for previously diagnosed HAT patients and were followed up in their communities. All these cases had been treated with Suramin I.V $20 \mathrm{mg} /$ $\mathrm{kg}$ body weight for 23 days and Melarsoprol I.V $3.6 \mathrm{mg} /$ $\mathrm{kg}$ body weight for 23 days for the early and late stage of HAT respectively according to Malawi HAT Treatment Guidelines (S1 Table). They were then followed up at 3, 6, and 12 months after discharge for review. The time between treatment and sample collection varied from a month to 48 months, but $25 \%$ of the samples were collected within the same year the individuals got their treatment.

The active screening was conducted during the follow-up of HAT cases, and nines cases were identified. After taking a history and an examination related to HAT infection, venous blood was collected in heparinized tubes and taken to the laboratory at a temperature of $4^{\circ} \mathrm{C}$. Eight capillary tubes were prepared from each sample and the buffy coat was examined by microscopy looking for trypanosomes. Two milliliters of whole blood was kept for DNA extraction. The remaining blood was used for plasma separation. Blood was centrifuged at 5000 revolution per minute (rpm) for 10 minutes in thermo scientific Legend Micro 17R centrifuge to separate plasma from the rest of the blood.

Using pasture pipettes (BRANDR India) the plasma was separated from red blood cells and stored in $-80^{\circ} \mathrm{C}$ freezer for cytokines analysis. The RBC was also stored in $-80^{\circ} \mathrm{C}$ freezer.

This study is part of a $\mathrm{PhD}$ project, which looks at the immunological and genetic factors that contribute to the pathogenesis of T.b. rhodesiense in Malawi. Out of 350 people screened, 243 individuals were enrolled into the study ${ }^{33,34}$. HAT cases were defined as individuals in whom trypanosomes were detected by microscopy in blood, lymphatic fluid or cerebral spinal fluids (CSF). HAT negative controls were defined as individuals with no signs and symptoms suggestive of HAT and no trypanosomes detected from the blood, lymphatic fluid or CSF.

Sample size for this study was calculated based on World Health Organization (WHO) indicated HAT data for Malawi of $41 \pm 13$ SD HAT cases annually ${ }^{2}$. To obtain $95 \%$ confidence interval at 5\% type 1 error $(\mathrm{p}<0.05)$, a minimum of 26 HAT cases and 26 controls are required to be included in the study ${ }^{35,36}$. For this study, 233 samples, 76 HAT cases and 157 controls were available for cytokine measurement and all 233 samples were included in the study.

HAT cases and controls came from the same area and lived in the same area from birth and had been exposed to infection since birth.

\section{Ethics statement}

The protocol was approved by the Malawi National Health Sciences Research Committee (NHSRC), protocol numbers NHSRC 15/4/1399 and Malawi 1213. There was also local involvement of all stakeholders, and local leaders gave approval for the study to be conducted in the area. All individuals enrolled in the study were 18 years of age or older. All individuals signed informed consent forms in their native language.

\section{Cytokines Enzyme-Linked Immunosorbent Assay (ELISA)}

Cytokine concentrations $\{$ TNF- $\alpha$, IL-8, IL-10, (Kit Cat. No. 555212, 555244 and 555157 respectively) $\}$ were measured using a solid phase sandwich Enzyme-Linked Immunosorbent Assay (ELISA) (BD OptiEIA, BD Biosciences PharMingen, San Diego, USA) according to the manufacturer's instructions (BD, Biosciences) and as described previously ${ }^{12,22}$. All assays were done in triplicate. The concentrations were read using a BioTek ${ }^{\text {TM }}$ ELx800 ${ }^{\text {TM }}$ Absorbance Microplate Reader and Gen5 v1.11 reader software (Bio'Tek Instruments, Inc., Winooski, VT, USA) as per manufacturer's instruction and as described previously ${ }^{12,22}$.

\section{Statistical analysis}

Demographic characteristics of the participants were summarized by frequency (percentage), mean and standard deviation while median (interquartile range [IQR]) was used for cytokines. Data distribution normality was analyzed with the Kolmogorov-Smirnov test. Chi-square was used to test for gender differences while age was tested using Student's T-test. Cytokine group differences were explored by MannWhitney U test. Correlation analysis was carried out using the Spearman correlation test for non-parametrically distributed variables and Pearson correlation for normally distributed variables. Data entry and sorting were carried out using Microsoft office Excel 2016. Statistical analysis was performed using IBM SPSS software for Windows, version 23 (IBM Corp., Armonk, N.Y., USA.), and we used R version 
Table 1: Demographic characteristics of all study participants-HAT cases and controls.

\begin{tabular}{|l|l|l|l|l|}
\hline Variable & All $(\mathrm{N}=233)$ & Cases $(\mathrm{N}=76)$ & Controls $(\mathrm{N}=157)$ & $\mathrm{P}$ value \\
\hline Age (years) $\mathrm{M} \pm \mathrm{SD}$ & $37.45 \pm 13.48$ & $39.9 \pm 13.9$ & $36.3 \pm 13.1$ & 0.281 \\
\hline Male gender $\mathrm{N}(\%)$ & $167(71.7)$ & $54(71.1)$ & $113(71.9)$ & 0.884 \\
\hline
\end{tabular}

$P$ values refer to Chi-square test, $p$-values $<0.05$ are in bold. $M$-mean, SD-Standard deviation, $N(\%)$-Number (percentage).

Table 2: Cytokine levels of all study participants-HAT patients and controls.

\begin{tabular}{|l|l|l|l|l|}
\hline Variable & All $(\mathrm{N}=233)$ & Cases $(\mathrm{N}=76)$ & Controls $(\mathrm{N}=157)$ & P value \\
\hline TNF- $\mathrm{a}(\mathrm{pg} / \mathrm{ml}) \mathrm{M} \pm \mathrm{SD}$ & $73.25 \pm 403$ & $29.35 \pm 181.58$ & $94.5 \pm 474.12$ & 0.033 \\
\hline $\mathrm{IL}-10(\mathrm{pg} / \mathrm{ml}) \mathrm{M} \pm \mathrm{SD}$ & $42.16 \pm 88.85$ & $44 \pm 81.7$ & $41.27 \pm 92.34$ & 0.734 \\
\hline $\mathrm{IL}-8(\mathrm{pg} / \mathrm{ml}) \mathrm{M} \pm \mathrm{SD}$ & $1910.48 \pm 4942.35$ & $1191.3 \pm 4236.09$ & $2258.6 \pm 5227.4$ & 0.002 \\
\hline
\end{tabular}

$P$ values refer to t test, $p$-values $<0.05$ are in bold. M-mean, SD-Standard deviation, $N(\%)$-Number (percentage), TNF- $\alpha-$ tumor necrosis factor alpha, IL-10-interleukin 10, IL-8-interleukin 8.

Table 3: Correlation of Demographic variables, and cytokine levels in all study participants-HAT patients and controls.

\begin{tabular}{|l|l|l|l|l|}
\hline & Age & TNF-a pg/ml & IL-10 pg/ml & IL-8 pg/ml \\
\hline TNF-a pg/ml & $0.066(0.323)$ & - & & \\
\hline IL-10 pg/ml & $0.025(0.708)$ & $0.215(0.001)$ & - & \\
\hline IL-8 pg/ml & $0.086(0.198)$ & $0.288(<0.001)$ & $0.110(0.094)$ & - \\
\hline
\end{tabular}

Correlation coefficient refers to Spearmans rho, $p$-values $<0.05$ are in bold. TNF- $\alpha$ - tumor necrosis factor alpha, IL-10interleukin 10, IL-8 - interleukin 8.

4.1.0 for data visualization. All reported $\mathrm{p}$-values are twosided, and significance was accepted at $\mathrm{p}<0.05$.

\section{Results}

Demographic characteristics and levels of plasma cytokines of all study participants-HAT cases and controls.

Two-hundred and thirty-three plasma samples were tested, and these included 76 HAT cases and 157 controls. Among the cases, nine had their plasma collected before treatment (were untreated) and the rest were treated before blood for plasma analysis was collected.

The mean age of HAT cases was higher than controls (Table 1). Seventy-one percent $(71 \%)$ of the participants in this study were male. Controls had significantly higher mean plasma levels of TNF- $\alpha(94.5 \pm 474.12 \mathrm{pg} / \mathrm{ml})$ and IL-8 $(2258.6 \pm 5227.4 \mathrm{pg} / \mathrm{ml}$, ) than HAT cases $(\mathrm{P}=0.033$ and $\mathrm{P}=0.002$, respectively). Mean plasma levels of IL-10 were similar between controls and patients. Cases represented $(33 \%)$ of all participants (Table 2$)$.

\section{Correlation levels of plasma cytokines and demographic variables of all study participants}

There was a statistically significant positive correlation between plasma TNF- $\alpha$ with IL-10 $(\mathrm{p}=0.001)$ and IL-8 $(\mathrm{p}<0.001)$ (Table 3). IL-10 and IL-8 were positively correlated but the relationship was not statistically significant $(\mathrm{p}=0.094)$.

\section{Effect of treatment on cytokine levels.}

We further examined the differences plasma cytokine levels between HAT cases who reported being on treatment before blood collection and those who were not treated before blood collection.

IL-10 median plasma levels were higher among untreated cases than treated cases; however, the difference was not statistically significant $(\mathrm{P}=0.073)$ as shown in Figure 1.

IL-8 median plasma levels were higher amongst HAT untreated cases than HAT treated cases and were statistically significant $(\mathrm{P}=0.006)$. For $\mathrm{TNF}-\alpha$ median levels, there is no statistically significant difference between the cases who were treated and those who were untreated $(\mathrm{P}=0.520)$.

\section{Discussion}

In the present study, plasma levels of IL-8, TNF- $\alpha$, and IL10 were quantified in HAT cases (treated and untreated) and controls. Our study showed statistically significant higher levels of IL-8 in plasma of untreated HAT cases than in treated HAT cases, as well as statistically significant higher levels of IL-8 in plasma of controls than in the HAT cases. IL-8 is a major cytokine that attract neutrophils during innate immune responses. In humans, over $50 \%$ of leukocytes are neutrophils, which produce antimicrobial peptides such as cathelicidins, known to be typanotoxic ${ }^{37}$. The high levels of IL- 8 in the untreated HAT cases is in line with the role of IL-8 of attracting neutrophils and mounting an innate immune response to infection after exposure ${ }^{31}$. This supports the findings by Lejon et al in T. b. gambiense infection conducted in DRC where it was found that the levels of IL-8 dropped after treatment ${ }^{29}$. Study of T. b. gambiense infection in West Africa showed that "trypanotolerant" (SERO TL+) subjects had elevated levels of IL-8, IL- 6 , and TNF- $\alpha$ than patients showing a stronger inflammatory response ${ }^{31}$.

TNF- $\alpha$ is a pro-inflammatory cytokine that is upregulated https://dx.doi.org/10.4314/mmj.v33i4.2 


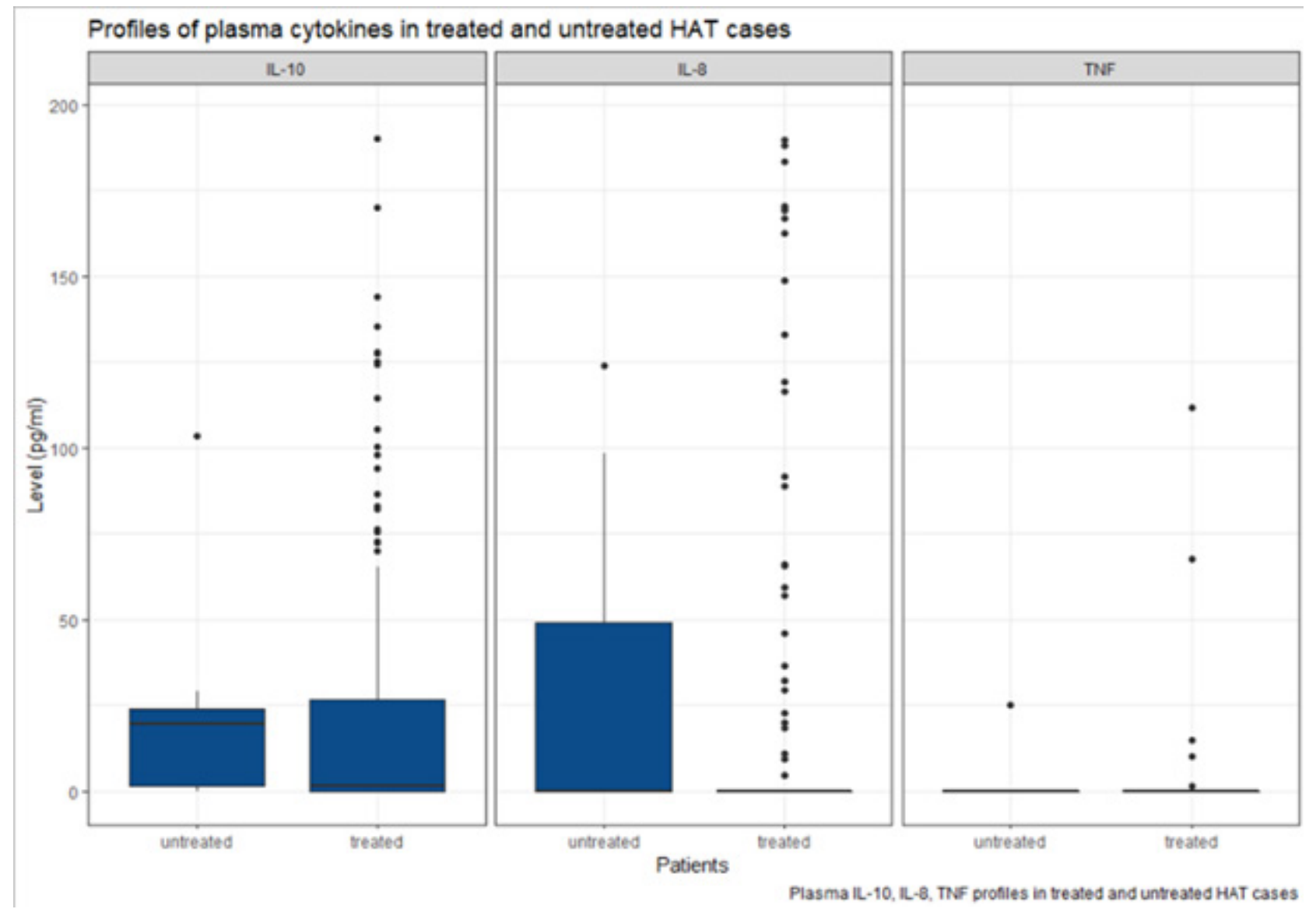

Figure 1. Profile of plasma cytokines in treated HAT cases and untreated HAT cases.

\section{Boxes represent the medians and interquartile ranges, and whiskers represent 10th and 90th percentiles}

during infection as described by Maina et $\mathrm{al}^{18}$. TNF- $\alpha$ is involved in the innate immunity and is produced by macrophages. Its production is induced by soluble VSGs shed by live trypanosomes ${ }^{38}$. It is known that TNF- $\alpha$ is produced during the first peak of parasitemia in the early stages of the infection ${ }^{39}$. TNF- $\alpha$ is involved in both parasitemia control and infection associated pathology during HAT infections ${ }^{28}$. Our data showed statistically significant higher levels of TNF- $\alpha$ in plasma of controls than the HAT cases, but there was no statistically significant difference in the levels of TNF- $\alpha$ in plasma between untreated and treated HAT cases. Contrary to our data, a study by Maclean et al, showed elevated levels of TNF- $\alpha$ in plasma in early stages of T. $b$. rhodesiense infections in Ugandan patients but were normal in the late stages of the infection ${ }^{11}$. The higher levels of TNF- $\alpha$ in Ugandan HAT cases was associated with rapid disease progression with increased disease severity compared to HAT cases in Malawi, who had low levels. In a similar study by Kato et al, TNF- $\alpha$ was only detected in plasma of two cases, which were early stage, but was not detectable in CNS (late stages) $^{11,12,29}$. In another related HAT study ${ }^{22}$, levels of TNF- $\alpha$ in plasma was low in cases and not significantly different from the controls.

IL-10 is a cytokine produced mainly by T-helper (Th) 2 cells ${ }^{40}$. IL-10 is known to play a major role in controlling both innate and adaptive immunity. Its main function is to limit and ultimately terminate inflammatory responses. IL-10 has been shown to protect the host in a number of parasitic diseases, including malaria, toxoplasmosis and in autoimmune encephalitis ${ }^{41,42}$. IL-10 down regulates the macrophages to become more anti-inflammatory type, thus controlling both the parasites and pathology $y^{43}$. Data from other studies have shown that IL-10 downregulates the production other cytokines such IFN- $\gamma$ and TNF- $\alpha$ by classically activated (M1) macrophages ${ }^{44-46}$. Our data shows the levels of IL-10 in plasma were similar in controls and HAT cases, whereas there were higher levels of IL-10 in plasma of untreated HAT cases than the treated HAT cases. However, the difference was not statistically significant. Previous studies have shown that in both T. b. rhodesiense and T. b. gambiense infections, levels of IL-10 in plasma are higher in cases than controls and that IL-10 levels in both plasma and CNS were upregulated particularly in the late stage in both T. b. rhodesiense and T. b. gambiense infections ${ }^{11,12}$, $29,47-49$.

Our study has several limitations. Because of the low disease incidence, our study depended on a limited number of samples especially for the untreated HAT cases, and this could prevent the study from finding evidence of small differences in cytokine levels. Furthermore, the inclusion of retrospective HAT cases might not give a true representation of cytokine profiles as some of the cases had already recovered from HAT (duration from time of treatment to sample collection was between a month to 48 months). The study did not screen for other protozoan infections such as malaria (as it was beyond the scope of this study), which may affect levels of the cytokines in plasma in individuals with coinfection $^{41,50,51}$.

\section{Conclusion}

In conclusion, this study gives a preliminary insight of cytokine profiles in HAT individuals in Malawi, which may be used as a basis for further investigation in the future. https://dx.doi.org/10.4314/mmj.v33i4.2 
Our data suggests that IL-8 could further be evaluated as biomarkers for HAT infection. Further studies with a larger cohort of cases (followed up during treatment), and additional cytokines which are associated with HAT infection outcomes would be useful in this area.

\section{Acknowledgement}

We would like thank the individuals that participated in this study, local leaders and National Control Programme (Trypanosomiasis) in the Ministry of Health, Malawi and Kamuzu University of Health Sciences and all colleagues who contributed to this work.

We are very grateful to the members of TrypanoGen Research Group for all the support including financial. Wellcome Trust funded this work through study number 099310/Z/12/Z.

\section{References}

1. Fèvre EM, Wissmann Bv, Welburn SC, Lutumba P. The Burden of Human African Trypanosomiasis. PLoS Negl Trop Dis 2008; 2 (12): e333. https://doi.org/10.1371/journal.pntd.0000333.

2. Franco J, Simarro P, Diarra A, Jannin J. Epidemiology of human African trypanosomiasis. Clin Epidemiol. 2014; 6 (1):257-275https:// doi.org/10.2147/CLEP.S39728

3. Courtin D, Berthier D, Thevenon S, Dayo GK, Garcia A, Bucheton B. Host genetics in African trypanosomiasis. Infect Genet Evol. 2008 May; 8 (3):229-38. doi: 10.1016/j.meegid.2008.02.007.

4. Simarro PP, Cecchi G, Franco JR, Paone M, Diarra A, Ruiz-Postigo JA, et al. Estimating and mapping the population at risk of sleeping sickness. PLoS Negl Trop Dis.2012; 6 (10): e1859.https://doi. org/10.1371/journal.pntd.0001859

5. Matemba LE, Fèvre EM, Kibona SN, et al. Quantifying the Burden of Rhodesiense Sleeping Sickness in Urambo District, Tanzania PLOS Negl Trop Dis. 2010 Nov; 4 (11): e868.DOI: 10.1371/journal. pntd.0000868. PMID: 21072230; PMCID: PMC2970539.

6. Odiit M, Coleman PG, Liu W-C, McDermott JJ, Fèvre EM, Welburn $\mathrm{SC}$, et al. Quantifying the level of under-detection of Trypanosoma brucei rhodesiense sleeping sickness cases. Trop Med Int Health 2005; 10 (9): 840-849. https://doi.org/10.1111/j.1365-3156.2005.01470.x

7. Barrett MP, Burchmore RJ, Stich A, Lazzari JO, frasch AC, Cazzulo JJ, et al. The trypanosomiases. Lancet 2003 Nov; 362 (9394): 1469 1480. https://doi.org/10.1016/S0140-6736(03)14694-6

8. Foulkes JR. The six diseases WHO. Human trypanosomiasis in Africa. Br Med J (Clin Res Ed). 1981 Oct; 283 (6300): 1172-1174. DOI: 10.1136/bmj.283.6300.1172. PMID: 6794806; PMCID: PMC1507339.

9. MacLean LM, Odiit M, Chisi JE, Kennedy PG, Sternberg JM. Focusspecific clinical profiles in human African Trypanosomiasis caused by Trypanosoma brucei rhodesiense. PLoS Negl Trop Dis. 2010 Dec; 4 (12): e906. DOI: 10.1371/journal.pntd.0000906. PMID: 21151878; PMCID: PMC2998431.

10. Kato CD, Nanteza A, Mugasa C, Edyelu A, Matovu E, Alibu VP. Clinical Profiles, Disease Outcome and Co-Morbidities among T. b. rhodesiense Sleeping Sickness Patients in Uganda. PLOS ONE 2015 Feb; 10 (2): e0118370. DOI: 10.1371/journal.pone.0118370. PMID: 25719539; PMCID: PMC4342333.

11. MacLean L, Chisi JE, Odiit M, Gibson WC, Ferris V, Picozzi K, et al. Severity of human african trypanosomiasis in East Africa is associated with geographic location, parasite genotype, and host inflammatory cytokine response profile. Infect Immun. 2004; 72 (12): 7040-7044. DOI: https://doi.org/10.1128/IAI.72.12.7040-7044.2004

12. MacLean L, Odiit M, MacLeod A, Morrison L, Sweeney L, Cooper A, et al. Spatially and Genetically Distinct African Trypanosome Virulence Variants Defined by Host Interferon- $\gamma$ Response. J Infect Dis. 2007 Dec; 196 (11): 1620-1628. https://doi.org/10.1086/522011
13. Dinarello CA. Proinflammatory cytokines. Chest 2000 Aug; 118 (2): 503-508. doi: 10.1378/chest.118.2.503. PMID: 10936147.

14. Sternberg JM, Maclean L. A spectrum of disease in human African trypanosomiasis: the host and parasite genetics of virulence. Parasitology 2010 Dec; 137 (14): 2007-2015.doi: 10.1017/S0031182010000946. Epub 2010 Jul 21. PMID: 20663245.

15. Janeway CA, Medzhitov R. Innate Immune Recognition. Annu Rev Immunol. 2002; 20 (1): 197-216. doi: 10.1146/annurev. immunol.20.083001.084359. Epub 2001 Oct 4. PMID: 11861602.

16. Shi M, Wei G, Pan W, Tabel H. Trypanosoma congolense infections: antibody-mediated phagocytosis by Kupffer cells. J Leukoc Biol.2004; 76 (2): 399-405. https://doi.org/10.1189/jlb.1003500

17. Coller SP, Mansfield JM, Paulnock DM. Glycosylinositolphosphate Soluble Variant Surface Glycoprotein Inhibits IFN- $\gamma$-Induced Nitric Oxide Production Via Reduction in STAT1 Phosphorylation in African Trypanosomiasis. J. Immunol. 2003 Aug ; 171 (3): 1466-1472.DOI: 10.4049/jimmunol.171.3.1466. PMID: 12874239.

18. Maina N, Ngotho JM, Were T, Thuita JK, Mwangangi DM, Kagira $\mathrm{JM}$ et al. Proinflammatory Cytokine Expression in the Early Phase of Trypanosoma brucei rhodesiense Infection in Vervet Monkeys (Cercopithecus aethiops). Infect Immun. 2004; 72 (5): 3063-3065. https://doi.org/10.1128/IAI.72.5.3063-3065.2004

19. Harris TH, Cooney NM, Mansfield JM, Paulnock DM . Signal Transduction, Gene Transcription, and Cytokine Production Triggered in Macrophages by Exposure to Trypanosome DNA. Infect Immun. 2006; 74 (8): 4530--4537. DOI: 10.1128/iai.01938-05. PMID: 16861639; PMCID: PMC1539588.

20. Mansfield JM, Paulnock DM. Regulation of innate and acquired immunity in African trypanosomiasis. Parasite Immunol. 2005 Oct; 27(10-11): 361-371.DOI: 10.1111/j.1365-3024.2005.00791.x. PMID: 16179030 .

21. Magez S, Radwanska M, Drennan M, Fick L, Baral TN, Brombacher $\mathrm{F}$, et al. Interferon-gamma and nitric oxide in combination with antibodies are key protective host immune factors during trypanosoma congolense Tc13 Infections. J Infect Dis. 2006 Jun; 193 (11): 15751583. https://doi.org/10.1086/503808

22. MacLean L, Odiit M, Sternberg JM. Nitric oxide and cytokine synthesis in human African trypanosomiasis. J Infect Dis. 2001 Oct; 184 (8): 1086-90.doi: 10.1086/323479. Epub 2001 Sep 28. PMID: 11574928 .

23. Namangala B, De Baetselier P, Beschin A. Both Type-I and Type-II Responses Contribute to Murine Trypanotolerance. J Vet Med Sci. 2009 Mar; 71 (3): 313-318.DOI: 10.1292/jvms.71.313. PMID: 19346699.

24. Uzonna JE, Kaushik RS, Gordon JR, Tabel H. Cytokines and antibody responses during Trypanosoma congolense infections in two inbred mouse strains that differ in resistance. Parasite Immunol. 1999 Feb; 21 (2): 57-71. doi: 10.1046/j.1365-3024.1999.00202.x. PMID: 10101716 .

25. Mertens B, Taylor K, Muriuki C, et al. Cytokine mRNA profiles in trypanotolerant and trypanosusceptible cattle infected with the protozoan parasite Trypanosoma congolense: protective role for interleukin-4? J Interferon Cytokine Res. 1999 Jan; 19 (1): 59-65. doi: 10.1089/107999099314423. PMID: 10048769.

26. Sternberg JM, Rodgers J, Bradley B, MacLean L, Murray M, Kennedy PGE. Meningoencephalitic African trypanosomiasis: Brain IL-10 and IL-6 are associated with protection from neuro-inflammatory pathology. J Neuroimmunol. 2005 Oct; 167 (1-2): 81-89. https://doi. org/10.1016/j.jneuroim.2005.06.017

27. Kennedy PGE. Cytokines in central nervous system trypanosomiasis: cause, effect or both? Trans R Soc Trop Med Hyg. 2009 Mar; 103 (3): 213-214. https://doi.org/10.1016/j.trstmh.2008.08.013

28. Magez S, Radwanska M, Beschin A, Sekikawa K, DeBaetselier P. Tumor Necrosis Factor Alpha Is a Key Mediator in the Regulation of 
Experimental Trypanosoma brucei Infections. Infect Immun. 1999; 67 (6): 3128-3132. DOI: https://doi.org/10.1128/IAI.67.6.3128-3132.1999

29. Lejon V, Lardon J, Kenis G, Pinoges L, Legros D, Bisser S, et al. Interleukin (IL)-6, IL-8 and IL-10 in serum and CSF of Trypanosoma brucei gambiense sleeping sickness patients before and after treatment. Trans R Soc Trop Med Hyg. 2002; 96 (3): 329-333. doi: . Epub 2002 0927

30. Bickel M. The role of interleukin- 8 in inflammation and mechanisms of regulation. J Periodontol 1993; 64 (5): 456-460. PMID: 8315568.

31. Ilboudo H, Bras-Gonçalves R, Camara M, Flori L, Camara O, Sakande H, et al. Unravelling Human Trypanotolerance: IL8 is Associated with Infection Control whereas IL10 and TNFa Are Associated with Subsequent Disease Development. PLOS Pathog. 2014 Nov 6; 10 (11): e1004469. doi: 10.1371/journal.ppat.1004469. PMID: 25375156; PMCID: PMC4223068.

32. Lemerani M, Jumah F, Bessell P, Biéler S, Ndung'u JM. Improved Access to Diagnostics for Rhodesian Sleeping Sickness around a Conservation Area in Malawi Results in Earlier Detection of Cases and Reduced Mortality. J Epidemiol Glob Health 2020; 10 (4): 280-287. https://doi.org/10.2991/jegh.k.200321.001

33. Ilboudo H, Noyes H, Mulindwa J, et al. Introducing the TrypanoGEN biobank: A valuable resource for the elimination of human African trypanosomiasis. PLoS Negl Trop Dis. 2017 Jun; 11 (6): e0005438. DOI: 10.1371/journal.pntd.0005438. PMID: 28570558; PMCID: PMC5453417.

34. Kamoto K, Noyes H, Nambala P, Senga E, Musaya J, Kumwenda B, et al. Association of APOL1 renal disease risk alleles with Trypanosoma brucei rhodesiense infection outcomes in the northern part of Malawi. PLoS Negl Trop Dis. 2019 Aug; 13 (8): e0007603.https://doi.org/ 10.1371/journal.pntd.0007603

35. Pourhoseingholi MA, Vahedi M, Rahimzadeh M. Sample size calculation in medical studies. Gastroenterol Hepatol Bed Bench 2013; 6 (1): 14-17 PMID: 24834239; PMCID: PMC4017493.

36. Metcalfe C. Biostatistics: A Foundation for Analysis in the Health Sciences. 7th edn. Wayne W. Daniel, Wiley, 1999. No. of. pages: xiv+755+appendices.. ISBN 0-471-16386-4. Stat Med. 2001; 20: 324 326.https://doi.org/10.1002/1097-0258(20010130)20:2<324::AIDSIM635>3.0.CO;2-O

37. Harrington JM. Antimicrobial peptide killing of African trypanosomes. Parasite Immunol. 2011; 33 (8): 461-469.doi:10.1111/ j.1365-3024.2011.01294.x

38. Magez S, Stijlemans B, Radwanska M, Pays E, Ferguson MA, DE Baetselier P. The glycosyl-inositol-phosphate and dimyristoylglycerol moieties of the glycosylphosphatidylinositol anchor of the trypanosome variant-specific surface glycoprotein are distinct macrophage-activating factors. J Immunol. 1998; 160 (4): 1949-1956. http://www.jimmunol. org/content/160/4/1949

39. Namangale B, De Baestselier P, Noël W, Brys L, Beschin A. Alternative versus classical macrophage activation during experimental African trypanosomosis. J Leukoc Biol. 2001; 69 (3): 387-396.https:// doi.org/10.1189/jlb.69.3.387
40. Amin DN, Vodnala SK, Masocha W, Sun B, Kristensson K, Rottenberg ME. Distinct Toll-like receptor signals regulate cerebral parasite load and interferon $\alpha / \beta$ and tumor necrosis factor $\alpha$-dependent T-cell infiltration in the brains of Trypanosoma brucei-infected mice. J Infect Dis. 2012; 205 (2): 320-332. https://doi.org/10.1093/infdis/ jir734

41. Hunt NH, Grau GE. Cytokines: accelerators and brakes in the pathogenesis of cerebral malaria. Trends Immunol. 2003 Sept; 24 (9): 491-499. https://doi.org/10.1016/S1471-4906(03)00229-1

42. Sarciron ME, Gherardi A. Cytokines involved in Toxoplasmic encephalitis. Scand J Immunol. 2000; 52 (6): 534-543. https://doi. org/10.1111/j.1365-3083.2000.00817.x

43. Namangala B, Noël W, De Baetselier P, Brys L, Beschin A. Relative contribution of interferon-gamma and interleukin-10 to resistance to murine African trypanosomosis. J Infect Dis. 2001; 183 (12): 1794 1800. DOI: $10.1086 / 320731$

44. Fiorentino DF, Zlotnik A, Mosmann TR, Howard M, O'Garra A. IL10 inhibits cytokine production by activated macrophages. J Immunol. 1991 Dec 1; 147 (11):3815-22. PMID: 1940369.

45. Guilliams M, Bosschaerts T, Hérin M, Hünig T, Loi P, Flamand $\mathrm{V}$, et al. Experimental expansion of the regulatory $\mathrm{T}$ cell population increases resistance to African trypanosomiasis. J Infect Dis 2008; 198 (5): 781-791. https://doi.org/10.1086/590439

46. Guilliams M, Movahedi K, Bosschaerts T, vanden Driessche T, Chuah M, Herin M, et al. IL-10 Dampens TNF/Inducible Nitric Oxide Synthase-Producing Dendritic Cell-Mediated Pathogenicity during Parasitic Infection. J Immunol. 2009; 182 (2): 1107-1118. DOI:10.4049/jimmunol.182.2.1107

47. MacLean L, Odiit M, Sternberg JM. Nitric oxide and cytokine synthesis in human African trypanosomiasis. J Infect Dis. 2001 Oct; 184 (8): 1086-1090.doi: 10.1086/323479. Epub 2001 Sep 28. PMID: 11574928 .

48. Courtin D, Jamonneau V, Mathieu J-F, Koffi M, Milet J, Yeminanga CS, et al. Comparison of cytokine plasma levels in human African trypanosomiasis. Trop Med Int Health 2006; 11 (5): 647-653. DOI:10.1111/j.1365-3156.2006.01612.x

49. Kato CD, Alibu VP, Nanteza A, Mugase CM, Matovu E. Interleukin (IL)-6 and IL-10 Are Up Regulated in Late Stage Trypanosoma brucei rhodesiense Sleeping Sickness. PLoS Negl Trop Dis. 2015; 9 (6): e0003835.https://doi.org/10.1371/journal.pntd.0003835

50. Kwiatkowski D, Sambou I, Twumasi P, Greenwood BM, Hill AVS, Manogue KR, et al. TNF concentration in fatal cerebral, nonfatal cerebral, and uncomplicated Plasmodium falciparum malaria. Lancet 1990; 336 (8725): 1201-1204.https://doi.org/10.1016/01406736(90)92827-5

51. Grau GE, Taylor TE, Molyneux ME, Wirima JJ, Vassalli P, Hommel M, et al. Tumor Necrosis Factor and Disease Severity in Children with Falciparum Malaria. N Engl J Med. 1989 Jun; 320 (24): 1586-91. DOI: 10.1056/NEJM198906153202404. PMID: 2657427. 